\title{
Different laterality indexes are poorly correlated with one another but consistently show the tendency of males and females to be more left- and right- lateralised, respectively
}

\author{
Carlos Buenaventura Castillo ${ }^{1,2}$, Andy Graeme Lynch $^{1,2}$ and Silvia Paracchini ${ }^{1 *}$ \\ ${ }^{1}$ School of Medicine, University of St Andrews, Scotland \\ ${ }^{2}$ School of Mathematics and Statistics, University of St Andrews, Scotland \\ *correspondence to: sp58@st-andrews.ac.uk
}

\begin{abstract}
Handedness is assessed primarily as a binary trait on the basis of the preferred hand for writing. At population level, about $90 \%$ people prefer using the right. Handedness can also be assessed as a continuous trait with laterality indexes, but these are not time and cost effective, and are not routinely collected. Here, we assessed the relationship of writing hand preference with four laterality indexes derived from measures of dexterity (pegboard task, marking squares and sorting matches) and strength (grip strength) available in a range of $\mathrm{N}=6664-8069$ children from the ALSPAC cohort. Although all indexes identified a higher proportion of individuals performing better with their right hand, they showed low correlation with each other (0.08-0.3). Analysis of sex effects on the laterality indexes showed that males and females tend to be, on all measures, more right- and the leftlateralised, respectively. Males also had a higher tendency to be poorly lateralised. This study shows that different handedness measures tap into different dimensions of laterality and cannot be used interchangeably. The similar trends for males and females observed across indexes suggest that sex effects should be taken into account in handedness and laterality studies.
\end{abstract}

Keywords: handedness, laterality, hand skills, behaviour, sex effect, ALSPAC 


\section{Introduction}

Worldwide, the vast majority of people (roughly 90\%) prefer using the right hand for most tasks in contrast to a minority of about $10 \%$ who prefer the left hand $(1,2)$. The rightward prevalence of handedness is a feature specific to humans. Language, which is unique to humans is also lateralised with a strong dominance for language processing in the left hemisphere. These observations have led to a number of studies investigating the role of handedness and brain asymmetries both in the context of human evolution and in cognitive abilities. Handedness has been investigated for association with cognitive skills, personality traits and psychiatric disorders (3-5). However, the cause/effect relationship between handedness, brain asymmetries and disorders remains unexplained and debated (6).

Intuitively, handedness is a binary category and most studies rely on the assignment of participants to a left/right-hand status based on preferred writing hand. Although a binary classification has many advantages, such as convenience and cost-effectiveness, it is has been argued that the preferred hand for writing is not a sensitive measure and does not capture a more general handedness assessment $(7,8)$. The Edinburgh Handedness Inventory (EHI) provides a solution by deriving a laterality quotient through hand preference scores on a series of tasks (9). These include items, such as brushing teeth, which are not expected to be influenced by cultural pressures. In fact, it is well established that environmental factors might force the use of the right hand for writing in people who might prefer using the left hand (10). This phenomenon was mainly true for past generations, and still applies in some cultures $(1,11)$. Typically, handedness questionnaires are characterised by a J-shaped distribution, indicating that the majority of people present an overall right- or left- hand preference with a few individuals in between.

Instead, relative hand skill tests lead to quantitative and continuous indexes of handedness. The indexes are derived by comparing performance of the two hands in carrying out skilled tasks. Most typically, these tests measure speed and dexterity, such as the Annett pegboard (12), marking squares and sorting matches tasks (13). The grip-strength test instead measures differences in manual strength (14). Test-retest correlations show that these tasks are sufficiently reliable $(15,16)$. Handedness indexes are derived by comparing the scores of the right (R) and left $(\mathrm{L})$ hand (see Methods) and lead to continuous distributions with positive means indicating a higher number of individuals who perform better with the right hand.

The higher frequency of better right-hand performance suggests there will a rough correlation across indexes. A difficulty in demonstrating this assumption is the availability of adequate datasets. While hand preference data are easy to collect in extremely large samples through self-reported 
questionnaire (17), performance tests are expensive and time-consuming. Therefore, collecting performance data in large samples is challenging, making it difficult to compare and evaluate correlations across tests. Multiple tasks available for the same participants are usually limited to a couple of measures as in the case of The National Child Development Study (NCDS) $(18,19)$ for which marking square and sorting matches tests were collected in over 6000 children (13). Only a few studies have examined the relationship across tests $(15,20)$.

Hand preference has been extensively analysed for the effect of sex, and males consistently show a higher prevalence of left-handedness (21). Although sex effects have been investigated in performance tasks, less focus has been given to laterality indexes. In general, females have been reported to perform faster with their favourite hand on the dexterity tests (22-25). Some of these effects have been suggested to be the results of smaller finger sizes in females compared to males, at least for the pegboard test $(22,26,27)$ but this interpretation is not consistently supported $(28)$. Instead, males tend to have higher scores than females in strength tests (29).

By taking advantage of the Avon Longitudinal Study of Parents and Children (ALSPAC), we aimed to address some of these issues around handedness measurements. We analysed different handedness indexes derived from the pegboard, marking squares, sorting matches and grip strength tasks in a range of 6664-8069 children. The indexes showed a higher proportion of children performing better with their right hand but were poorly correlated with one another. Consistently with previous literature, we found a higher frequency of left-handedness in males. Sex effects on the handedness indexes showed that male and females tend to be more left- and right-lateralized, respectively. These data show that different handedness measures are not interchangeable but are similarly influenced by sex.

\section{Material and Methods Samples}

The ALSPAC cohort.

ALSPAC is a longitudinal cohort representing the general population living in the Bristol area. The ALSPAC cohort consists of over 15,000 children from the southwest of England that had expected dates of delivery between 1st April 1991 and 31st December 1992 (30, 31). From age 7, all children were invited annually for assessments on a wide range of physical, behavioural, and neuropsychological traits, including cognitive (reading and mathematics related) measures. Attendance to the annual assessment determined the availability of data for the measures used in this study. Informed written consent was obtained from the parents after receiving a complete description 
of the study at the time of enrolment into the ALSPAC project, with the option for them or their children to withdraw at any time. Ethical approval for the study was obtained from the ALSPAC Law and Ethics Committee and the Local Research Ethics Committees.

\section{Phenotypes}

Self-reported hand preference for writing was collected at age seven.

The pegboard task was conducted as part of the movement assessment battery for children (Movement ABC; (32)). The child had to insert twelve pegs, one at a time, into a peg board, holding the board with one hand and inserting the pegs with the other, as quickly as possible. The task was carried out with the preferred and the non-preferred hand, after it had been described and demonstrated by the tester, and after a practice with each hand. The score corresponded to the time taken to complete the task with each hand.

The marking Square and Sorting Matches tasks are a repetition of those used in the NCDS (33) and were collected at age 10. Both tasks were first demonstrated by the tester and then the child had a practice. Scores were then collected from two measurements for each hand.

In the marking square task, the child is asked to make a short dash with a pencil on a piece of paper which has a grid made of rows of 20 squares. They are asked to start at the top left-hand side of the squared paper and move across it. When the first line is completed the child should move on the left side of the next row. The score corresponded to the number of squares that could be marked in 60 seconds and it was derived from the mean score of the two trials.

In the sorting matches task, the child is asked to move one match at a time across two boxes, one full and one empty using one hand only. The score corresponded to the time taken to transfer all the matches from one box to the other.

Grip strength was assessed with a Jamar hand dynamometer at age 11. After one demonstration from the tester, the child was given the opportunity to practice. The child was encouraged to squeeze the apparatus as long and as strongly as possible. The measurements were taken alternating hands for three times and starting with the right hand. The higher the reading (measured in kilograms), the stronger the grip. The mean from the three measurements for each hand was used. 
Please note that the ALSPAC website contains details of all the data that is available through a fully searchable data dictionary and variable search tool" and reference the following webpage http://www.bristol.ac.uk/alspac/researchers/our-data/

When scores for performance tasks were recorded for the "dominant/non-dominant" hand, we used information about the preferred hand for writing to define the scores for the left and right hand. When multiple trials were available a mean score across the trials was used. For all four performance tasks, we derived laterality indexes, namely PegQ, MarkQ, SOrtQ and GripQ, based on previous literature so that positive and negative scores corresponded to a better performance with the right and left hand, respectively(34, 35).

\section{Data analysis}

All analyses and data visualization were performed using the tidyverse and rlang packages R Studio v.3.5.1 (36). All analyses scripts are available through Open Science Framework https://osf.io/4ysnk/.

\section{Data availability}

Data used for this submission will be made available on request to the Executive (alspacexec@bristol.ac.uk). The ALSPAC data management plan (http://www.bristol.ac.uk/alspac/researchers/data-access/documents/alspac-data-managementplan.pdf) describes in detail the policy regarding data sharing, which is through a system of managed open access.

\section{RESULTS}

Handedness indexes

Analysis was conducted using data from the ALSPAC cohort. We assessed the frequency of hand preference for writing recorded as a self-reported measure when children were 7 years old ( $N=8069$, Table 1). In total, 977 (12.1\%) were left-handed. Consistently with previous literature (21), there was a higher proportion of left-handed males (13.6\%) than females (10.5\%). This measure did not identify any ambidextrous individuals. 
Table 1: Distribution of hand preference by gender

\begin{tabular}{cccc}
\hline & Right & Left & Total \\
\hline \multirow{2}{*}{ Male } & 3,530 & 558 & 4 \\
& $(86.4 \%)$ & $(13.6 \%)$ & 4,088 \\
Female & 3,562 & 419 & 3,981 \\
& $(89.5 \%)$ & $(10.5 \%)$ & \\
\multirow{2}{*}{ Total } & 7,092 & 977 & 8,069 \\
& $(87.9 \%)$ & $(12.1 \%)$ & \\
\hline
\end{tabular}

Four laterality indexes (PegQ, SortQ, MarkQ and GripQ) were derived from manual tasks that measure the performance with both the right $(R)$ and left $(L)$ hand. For all indexes a positive value indicates a better performance with the right hand (Table 2).

MarkQ presents a bimodal distribution, consistent with previous reports (13), while the others are unimodal and well-approximated by a normal distribution (Table 2, Supplementary Fig S1). All indexes are leptokurtic and have a positive mean, indicating that the majority of individuals performed better with the right hand. PegQ and SortQ exhibit discrete behaviour near zero as expected from their construction (See Supplementary Figure S1). MarkQ presented 13\% negative scores, similar to the frequency of individuals who preferred writing with the left hand. The other indexes had a larger proportion of negative scores (24\%-32\%; Table 2$)$.

Most data were collected within a period of roughly three months around the target age for each test but the actual age range spanned across two years (Table 2; Supplementary Figure S2). There was no age effect on the indexes (Supplementary Figure S2) and no age difference between males and females (Supplementary Table S1).

Table 2. Laterality indexes

\begin{tabular}{|c|c|c|c|c|c|c|c|c|c|c|}
\hline \multirow[b]{2}{*}{ Index } & \multirow[b]{2}{*}{ Formula } & \multirow[b]{2}{*}{$\mathbf{N}$} & \multicolumn{2}{|c|}{ Age (months) } & \multirow{2}{*}{$\begin{array}{c}\mathrm{N} \text { trials/ } \\
\text { hand }\end{array}$} & \multicolumn{5}{|c|}{ Index distribution } \\
\hline & & & Mean & SD & & Mean & SD & Skew & $\begin{array}{c}\text { Excess } \\
\text { Kurtosis }\end{array}$ & $\begin{array}{c}\% \text { negative } \\
\text { scores }\end{array}$ \\
\hline PegQ & $100 *(L-R) /(L+R)$ & 6884 & 92.3 & 3.9 & 1 & 5.37 & 9.81 & -0.19 & 0.35 & 24.16 \\
\hline MarkQ & $100 *(R-L) /(L+R)$ & 7389 & 130.1 & 3.2 & 2 & 13.95 & 13.59 & -0.65 & 0.96 & 13.51 \\
\hline SortQ & $100 *(L-R) /(L+R)$ & 7366 & 130.1 & 3.2 & 2 & 2.58 & 7.13 & -0.07 & 0.18 & 32.65 \\
\hline GripQ & $100 *(R-L) /(L+R)$ & 6664 & 143.4 & 2.9 & 3 & 3.41 & 6.33 & 0.48 & 5.25 & 25.32 \\
\hline
\end{tabular}

Age was reported in days (as in Supplementary Table S1), but presented here as months for easier interpretation. See Supplementary Figure S1 for the distribution of the indexes and Supplementary Figure 52 for age effects on the indexes 


\section{Correlation across measures}

We assessed the joint distributions of the indexes graphically, and with the Pearson correlation coefficient (Figure 1). In general, the correlations between indexes were low, ranging from 0.08 (SortQ-GripQ) to 0.3 (MarQ-PegQ). MarkQ was the index that best predicted the preferred hand for writing. This effect was also reflected by the bimodal distribution of MarkQ which separates individuals with a right and left writing preference. While not obviously bimodal, PegQ is the secondbest index at separating left and right writing preference. SortQ and GripQ do not effectively separate the populations. There was not substantial difference in this pattern when the analysis was conducted for male and females separately (Supplementary Figure S3). 


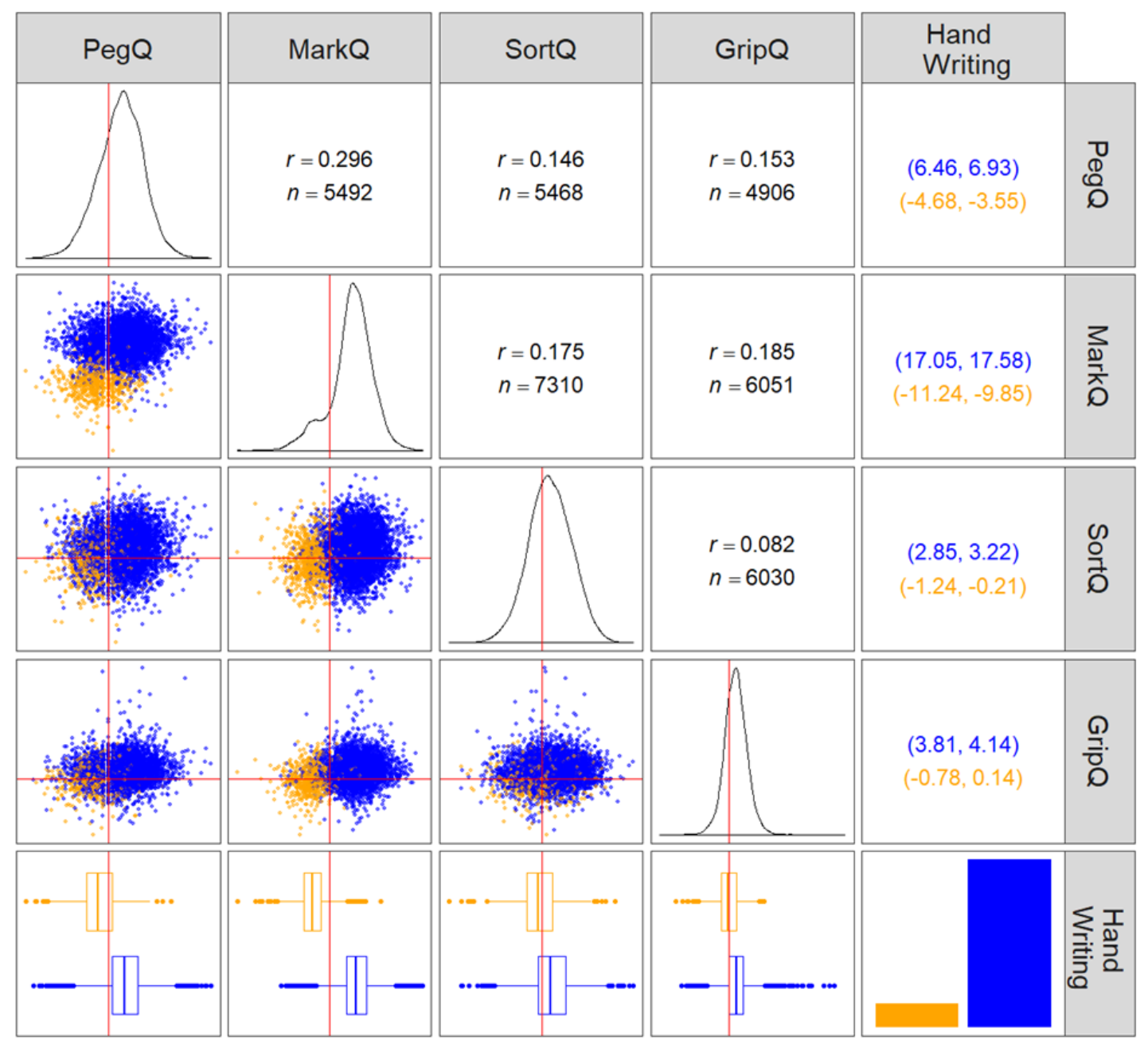

Figure 1. Correlation across la laterality measures. The cells along the diagonal show the empirical distribution for each index and, in the last box, the bar-plot for the preferred hand for writing. The boxes on the left of the main diagonal show the bivariate distribution of the indexes colour coded for preferred hand for writing (left = orange; right $=$ blue). For example, the first box on the second raw of the matrix illustrates the PegQ scores on the $x$-axis and MarkQ on the $y$-axis. The bottom row shows the box-plots for each laterality index grouped by preferred hand for writing. The red lines are aligned along the zero for each index. The cells on the right of the diagonal show the correlation coefficients and the sample sizes from which they were calculated. The last column reports the confidence intervals for the box-plots shown in the bottom row, providing a measure of how the different indexes separate the right and left handers for preferred writing hand. 
The structure amongst indexes was further explored with principal component analysis (PCA; Table 3 and Figure 2). The analysis was performed on 4,569 individuals who had no missing data for the four indexes as well as for the preferred hand for writing. The first component, PC1, explaining $38.7 \%$ of the variation, gives broadly equal weight to each variable, suggesting that, even though the correlations between the variables are not strong, they are all measuring the same underlying trait (handedness) and indeed this component shows discrimination between the left and right preferred hand for writing.

The remaining three components explain similar proportions of variance, and so may be somewhat arbitrarily ordered. This suggests that in addition to the common trait, each index is capturing a different characteristic. The components can be interpreted as a contrast between SortQ and GripQ (PC2), a contrast between PegQ and MarkQ (PC4), and a contrast between a combined PegQ/MarkQ and a combined SortQ/GripQ (PC3). Perhaps unexpectedly, given the results shown in Figure 1, PC3 does not greatly discriminate between left and right preference for writing hand (Figure 2).

Table 3. Principal Component Analysis

\begin{tabular}{|c|c|c|c|c|c|}
\hline & & PC1 & PC2 & PC3 & PC4 \\
\hline \multicolumn{2}{|c|}{ Standard deviation } & 1.244 & 0.962 & 0.914 & 0.831 \\
\hline \multicolumn{2}{|c|}{ Proportion of Variance } & 0.387 & 0.232 & 0.209 & 0.173 \\
\hline \multicolumn{2}{|c|}{ Cumulative Proportion } & 0.387 & 0.619 & 0.828 & 1.000 \\
\hline \multirow{4}{*}{ Loadings } & PegQ & 0.552 & 0.009 & 0.579 & 0.601 \\
\hline & MarkQ & 0.593 & -0.016 & 0.234 & -0.770 \\
\hline & SortQ & 0.411 & 0.719 & -0.544 & 0.136 \\
\hline & GripQ & 0.419 & -0.695 & -0.561 & 0.166 \\
\hline
\end{tabular}



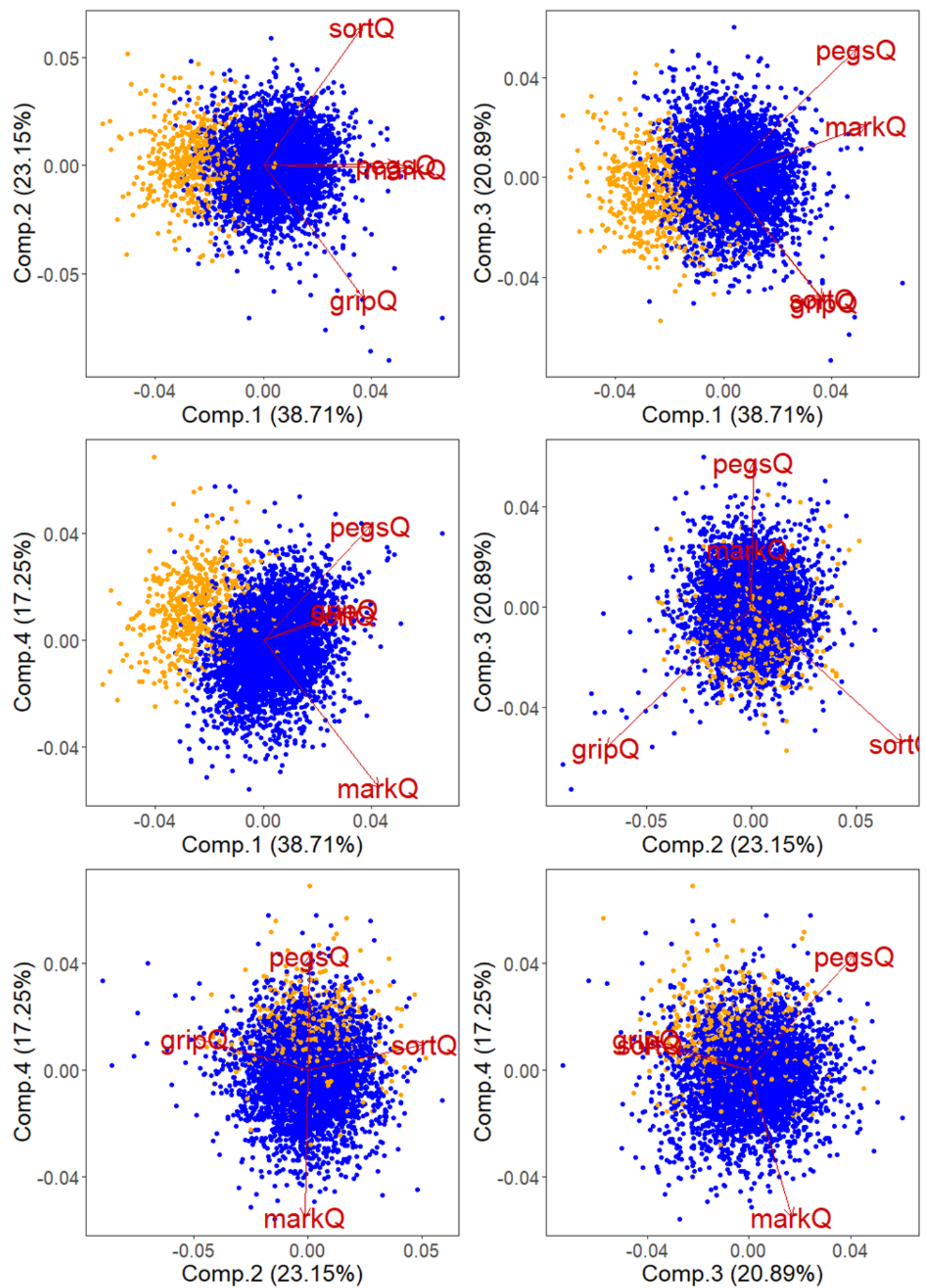

Figure 2. Biplots for all the PCA pairs. Each biplot visualises the contribution of all indexes to a principal component pair. The length of the arrows illustrates the variable contribution to each principal component. The colours indicate the preferred hand for writing for each individual: orange = left and blue = right. 


\section{Sex effects}

For each task, we first analysed gender effects on performance regardless of laterality, using a second independent two-sample t-test (Table 4). We used the best score regardless of which hand was used as a measure of performance (See supplementary Figure S4 to visualise the performance of the left versus right hand). We found significant differences between males and females in all the tasks $(p<0.0001)$. Females outperformed males in the pegboard, marking squares and sorting matches tasks, whereas males performed better in the grip strength task. Although age had an effect on performance, especially for grip strength (Pegboard: $r=-0.13, p<0.0001$; Marking squares: $r=$ $0.018, p=0.13$; Sorting matches: $r=-0.055, p<0.0001$; Grip strength: $r=0.15, p<0.0001$ supplementary Figure S5) there was no age difference between males and females (Supplementary Table 1) that could explain these sex effects.

Table 4 Gender differences in performance of the best hand

\begin{tabular}{|c|c|c|c|c|c|c|c|c|}
\hline \multirow{2}{*}{$\begin{array}{l}\text { Task } \\
\text { (unit) }\end{array}$} & \multicolumn{3}{|c|}{ Males } & \multicolumn{3}{|c|}{ Females } & \multirow{2}{*}{$\begin{array}{c}\text { Welch } \\
\text { t-test } \\
\text { P-val }\end{array}$} & \multirow{2}{*}{$\begin{array}{l}\bar{x}_{m}-\bar{x}_{f} \\
95 \% \text { C.I. }\end{array}$} \\
\hline & $\boldsymbol{n}_{m}$ & $\bar{x}_{m}$ & $s e_{m}$ & $n_{f}$ & $\bar{x}_{f}$ & $s e_{m}$ & & \\
\hline $\begin{array}{l}\text { Pegboard } \\
\text { (seconds) }\end{array}$ & 3,465 & 22.35 & 0.06 & 3,419 & 21.29 & 0.05 & $<0.0001$ & $(0.91,1.21)$ \\
\hline $\begin{array}{l}\text { Marking Squares } \\
\text { (marked squares) }\end{array}$ & 3,630 & 77.62 & 0.39 & 3,759 & 84.07 & 0.41 & $<0.0001$ & $(-7.56,-5.34)$ \\
\hline $\begin{array}{l}\text { Sorting matches } \\
\text { (seconds) }\end{array}$ & 3,627 & 39.35 & 0.11 & 3,739 & 37.84 & 0.10 & $<0.0001$ & $(1.22,1.8)$ \\
\hline $\begin{array}{l}\text { Grip strength } \\
\text { (kilograms) }\end{array}$ & 3,271 & 19.43 & 0.07 & 3,393 & 18.30 & 0.07 & $<0.0001$ & $(0.94,1.34)$ \\
\hline
\end{tabular}

$\overline{\boldsymbol{x}}$ = sample mean of the best hand performance; $\frac{\boldsymbol{s}_{x}}{\sqrt{n}}=$ standard deviation of the sample mean

Better performance is represented by a lower score for pegboard and sorting matches, and a higher score for marking squares and grip strength.

$\bar{x}_{m}-\bar{x}_{f} 95 \%$ C.I. Confidence intervals for the difference of the means in males and females.

Given these differences, and the higher frequency of left-hand preference in males compared to females (Table 1), we assessed sex effects on the laterality indexes. Comparison of the means of the distributions consistently showed a shift towards the left and right for males and females, respectively (Table 5). Of the principal components, only PC1, which captures a general laterality trait, shows strong differences between the sexes. The remaining components show little difference, suggesting that the indexes do not vary substantially according to sex. 
Table 5 Comparison of index means in males and females

\begin{tabular}{|c|c|c|c|c|c|c|c|c|}
\hline & \multicolumn{3}{|c|}{ Males } & \multicolumn{3}{|c|}{ Females } & \multirow{2}{*}{$\begin{array}{l}\text { Welch } \\
\text { t-test } \\
\text { P-val }\end{array}$} & \multirow{2}{*}{$\begin{array}{l}\bar{x}_{m}-\bar{x}_{f} \\
95 \% \text { C.I. }\end{array}$} \\
\hline & $n_{m}$ & $\bar{x}_{m}$ & $s e_{m}$ & $n_{f}$ & $\overline{\boldsymbol{x}}_{\boldsymbol{f}}$ & $s e_{f}$ & & \\
\hline PegsQ & 3,465 & 4.80 & 0.16 & 3,419 & 5.95 & 0.17 & $<0.0001$ & $(-1.61,-0.69)$ \\
\hline MarkQ & 3,630 & 13.09 & 0.23 & 3,759 & 14.79 & 0.21 & $<0.0001$ & $(-2.32,-1.08)$ \\
\hline SortQ & 3,627 & 1.88 & 0.12 & 3,739 & 3.26 & 0.11 & $<0.0001$ & $(-1.7,-1.05)$ \\
\hline GripQ & 3,271 & 3.17 & 0.11 & 3,393 & 3.65 & 0.11 & 0.002 & $(-0.78,-0.17)$ \\
\hline PC1 & 2,216 & -0.12 & 0.03 & 2,353 & 0.11 & 0.03 & $<0.0001$ & $(-0.3,-0.16)$ \\
\hline PC2 & 2,216 & -0.03 & 0.02 & 2,353 & 0.03 & 0.02 & 0.0231 & $(-0.12,-0.01)$ \\
\hline PC3 & 2,216 & 0.03 & 0.02 & 2,353 & -0.03 & 0.02 & 0.051 & $(0,0.11)$ \\
\hline PC4 & 2,216 & -0.01 & 0.02 & 2,353 & 0.01 & 0.02 & 0.6637 & $(-0.06,0.04)$ \\
\hline
\end{tabular}

$\overline{\boldsymbol{x}}$ = sample mean of the laterality index; $\frac{s}{\sqrt{n}}=$ standard deviation of the sample mean $\boldsymbol{s} \boldsymbol{e}=\frac{\boldsymbol{s}}{\sqrt{\boldsymbol{n}}}$, standard error

$\bar{x}_{m}-\bar{x}_{f} 95 \%$ C.I. Confidence intervals for the difference of the means in males and females. C.I. that do not overlap zero, as in the case for all indexes, provide evidence that the distributions are different between the two sexes.

To further assess sex effects on the indexes, we analysed the males/females ratios along the distributions. The ratios consistently decreased from the negative to positive scores for all indexes (Figure 3). This observation indicates an over-representation of males and females in left- and rightlateralised individuals, respectively. SortQ showed the most linear gradient with the most extreme values ranging from a male/female ratio of 1.43 in the most left-lateralized decile and of 0.77 in the most right-lateralized decile.

A

\begin{tabular}{ccccc}
\hline \multicolumn{5}{c}{ Male/Female ratio } \\
\hline Decile & PegQ & Marka & Sorta & GripQ \\
$0-10$ & 1.05 & 1.26 & 1.43 & 1.11 \\
$10-20$ & 1.09 & 1.43 & 1.26 & 1.11 \\
$20-30$ & 1.34 & 1.07 & 1.11 & 1.16 \\
$30-40$ & 1.12 & 0.93 & 1.09 & 0.95 \\
$40-50$ & 1.13 & 0.99 & 1.03 & 0.99 \\
$50-60$ & 0.96 & 0.86 & 0.99 & 1.04 \\
$60-70$ & 0.95 & 0.87 & 0.92 & 0.96 \\
$70-80$ & 0.84 & 0.85 & 0.84 & 0.98 \\
$80-90$ & 0.90 & 0.94 & 0.74 & 0.95 \\
$90-100$ & 0.74 & 0.94 & 0.77 & 0.79 \\
\hline
\end{tabular}

B

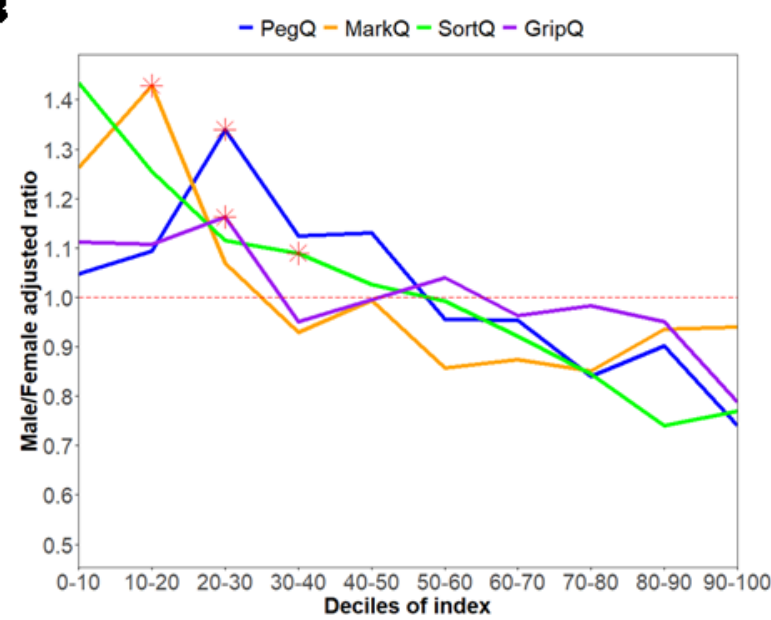

Figure 3. Males/females ratio across the distribution of the laterality indexes. The male/female ratio are shown as values (A) and visualised as graph (B). Each value has been corrected for the overall male/female ratio of data available for each index, i.e. $\mathrm{PegQ}=1.0135$ (=3465M/3419F); $\mathrm{MarkQ}=0.9657(=3630 \mathrm{M} / 3759 \mathrm{~F})$; SortQ $=0.9701(=3627 \mathrm{M} / 3739 \mathrm{~F}) ; \mathrm{GripQ}=0.964(3271 \mathrm{M} / 3393 \mathrm{~F})$. The deciles including scores $=0$ (i.e. ambidextrous on the corresponding task) are indicated in bold in (A) and with red asterisks in (B). The graph visualises the consistent tendency for males and females to be more left- and right-lateralised, respectively. 


\section{DISCUSSION}

We analysed different handedness-related measures testing specifically how they correlate with each other and how they are influenced by sex. We analysed four laterality indexes (PegQ, MarkQ, SortQ and MarkQ) and hand preference for writing in the ALSPAC dataset.

All indexes showed a majority of individuals performing better with the right hand (Table 2), but presented only a moderate correlation with hand preference for writing (Figure 2). MarkQ was the only bimodal index and best separated the individuals with a left- and right- hand preference for writing. The marking squares test is based on the use of a pen, therefore it is possible that the proficiency acquired for the preferred writing hand might influence this laterality index. Marking squares data were collected at age 10, when the preferred hand for writing is well established and therefore one hand would be more skilled than the other at using a pen. Conversely, the other tasks are expected to be less influenced by this kind of training. For example, sorting matches and moving pegs are not daily activities on which an individual would develop consistent exposure as opposed to holding a pen. After MarkQ, PegQ was the best measure at separating individuals for their preferred hand for writing. PegQ and MarkQ were the two indexes showing the highest correlation, which was however quite modest $(r=0.3)$.

Principal component analysis confirmed that the four indexes are necessary to capture the structure of the data (Table 3 and Figure 3), suggesting no redundancy of indexes. These data show that it remains a challenge to comprehensively assess handedness and to reduce such assessment in a single measure. For example, the poor correlation between indexes indicates that the generation of an handedness factor score does not seem a reliable option. Our analysis also implies that comparisons across handedness studies that used different handedness measures are not a straightforward process. In light of our current results, we strongly recommend to avoid referring to handedness as a generic measure or a universal concept, and encourage instead to refer to the specific tasks used for handedness assessment. Our results also raise the question of whether different laterality phenotypes are underlined by shared biology. For example, the most recent GWAS for hand preference highlighted that the biological pathways implicated in handedness also contribute to disorders such as schizophrenia and appear to be mediated by mechanisms controlling the cell cytoskeleton (4). Although associations were reported for different genes, previous genetic studies for continuous measures, and PegQ in particular, also suggested an overlap between the biology handedness and neurodevelopmental disorders and a role of cilia biology, a process closely link with cytoskeleton dynamics $(3,37-40)$. 
As reported in previous literature (21), we found a higher frequency of males who preferred writing with the left hand compared to females (Table 1). Also consistently with previous literature, we found that females outperformed males in dexterity tasks (pegboard, marking squares and sorting matches) but not strength (grip strength) tasks $(24,25,29)$ (Table 4). Some studies suggested that higher dexterity in females could be influenced by finger size (27) but these observations were limited to the pegboard task and failed to replicate in subsequent studies (28). We did not have measures of finger sizes in our dataset and therefore could not test this effect directly. However, we report better performance in females on the marking squares task as well, which is unlikely to be affected by finger size. Therefore, our data are indeed suggestive of higher dexterity in females.

Given the male/female differences in performance, we looked at sex effects on the laterality indexes. We found that males and females were more left- and right-lateralised, respectively, both in the dexterity- and strength-derived tasks (Table 5). The males/females ratio was $>1$ around the zero of the all distributions indicating a higher tendency for males to have similar scores for both hands or, in other words, to be poorly lateralized. To the best of our knowledge, the tendency for males and females to be over-represented at the opposite ends of the distributions of laterality indexes has not been reported before. Previous studies, showed that females had bigger differences between the preferred and not preferred hand on dexterity performance (22-25). However, if this was the case, we would expect an over-representations of females at both the left and right extremes. Instead, our analysis shows that females were over-represented only on the right side of the distribution for all indexes. While the majority of individuals had the similar age when performing the different tasks, the age range varied around a window of about two years. We ruled out any potential age effects that could explain the sex effect on the indexes (Supplementary Table S1).

In summary, we investigated different laterality measures in a large dataset. In agreement with previous literature, our results show a higher frequency of left-handedness in males and better dexterity performance in females. In addition, we report for the first time that males and females are more left- and right-lateralised on both dexterity and strength tasks. These data indicate the importance of factoring sex into any handedness and laterality study. The correlation across different indexes is weak, showing that different handedness tasks measure different handedness components and cannot be directly compared or combined and interpreted under a general handedness label. Overall, this work provides a reference for the design and interpretation of handedness studies especially when for the use of different measures. 


\section{Acknowledgements}

SP is a Royal Society University Research Fellow. The UK Medical Research Council and Wellcome (Grant ref: 102215/2/13/2) and the University of Bristol provide core support for ALSPAC. This publication is the work of the authors and Silvia Paracchini will serve as guarantor for the contents of this paper. A comprehensive list of grants funding is available on the ALSPAC website (http://www.bristol.ac.uk/alspac/external/documents/grant-acknowledgements.pdf). We are extremely grateful to all the families who took part in this study, the midwives for their help in recruiting them, and the whole ALSPAC team, which includes interviewers, computer and laboratory technicians, clerical workers, research scientists, volunteers, managers, receptionists and nurses. We are grateful to Judith Schmitz for useful comment to the manuscript.

\section{References}

1. Papadatou-Pastou M, Martin M, Munafo M, Ntolka E, Ocklenburg S, Paracchini S (2019): The prevalence of left-handedness: Five meta-analyses of 200 studies totaling 2,396,170 individuals. . doi: 10.31234/OSF.IO/5GJAC.

2. McManus IC (2002): Right hand, left hand. London, UK: Phoenix.

3. Brandler WM, Paracchini S (2014): The genetic relationship between handedness and neurodevelopmental disorders. Trends Mol Med, 2013/11/28. 20: 83-90.

4. Wiberg A, Ng M, Al Omran Y, Alfaro-Almagro F, McCarthy P, Marchini J, et al. (2019): Handedness, language areas and neuropsychiatric diseases: insights from brain imaging and genetics. Brain. . doi: $10.1093 /$ brain/awz257.

5. Paracchini S, Diaz R, Stein J (2016): Advances in Dyslexia Genetics-New Insights Into the Role of Brain Asymmetries. Adv Genet. 96: 53-97.

6. Bishop DVM (2013): Cerebral asymmetry and language development: cause, correlate, or consequence? Science (80- ), 2013/06/15. 340: 1230531.

7. Paracchini S, Scerri T (2017): Genetics of human handedness and laterality. In: Rogers L, Vallortigara G, editors. Lateralized brain Funct. Springer, pp 523-552.

8. Scharoun SM, Bryden PJ (2014): Hand preference, performance abilities, and hand selection in children. Front Psychol. 5: 82.

9. Oldfield RC (1971): The assessment and analysis of handedness: the Edinburgh inventory. Neuropsychologia, 1971/03/01. 9: 97-113.

10. Porac C, Coren S, Searleman A (1986): Environmental factors in hand preference formation: Evidence from attempts to switch the preferred hand. Behav Genet. 16: 251-261.

11. de Kovel CGF, Carrión-Castillo A, Francks C (2019): A large-scale population study of early life factors influencing left-handedness. Sci Rep. 9: 584.

12. Annett M (1972): The distribution of manual asymmetry. Br J Psychol, 1972/08/01. 63: 343-358.

13. McManus IC (1985): Right- and left-hand skill: failure of the right shift model. Br J Psychol. 76 ( Pt 1): 1-34.

14. Provins KA, Magliaro J (1993): The Measurement of Handedness by Preference and Performance Tests. Brain Cogn. 22: 171-181.

15. McManus IC, Van Horn JD, Bryden PJ (2016): The Tapley and Bryden test of performance differences between the hands: The original data, newer data, and the relation to pegboard and other tasks. Laterality Asymmetries Body, Brain Cogn. 21: 371-396. 
16. Annett M, Hudson PTW, Turner A (1974): The reliability of differences between the hands in motor skill. Neuropsychologia. 12: 527-531.

17. Kovel CGF de, Francks C (2018): The molecular genetics of hand preference revisited. bioRxiv. 447177.

18. Davie R, Butler N, Goldstein H (1972): From birth to seven: the second report of the national child development study. (1958 Cohort)). London Longmans. .

19. Power C, Elliott J (2006): Cohort profile: 1958 British birth cohort (National Child Development Study). Int J Epidemiol. 35: 34-41.

20. Annett M (1992): Five Tests of Hand Skill. Cortex. 28: 583-600.

21. Papadatou-Pastou M, Martin M, Munafò MR, Jones G V. (2008): Sex differences in lefthandedness: A meta-analysis of 144 studies. Psychol Bull. 134: 677-699.

22. Bryden PJ, Roy EA (2005): A new method of administering the Grooved Pegboard Test: Performance as a function of handedness and sex. Brain Cogn. 58: 258-268.

23. Bornstein RA (1985): Normative data on selected neuropsychological measures from a nonclinical sample. J Clin Psychol. 41: 651-659.

24. Thompson LL, Heaton RK, Matthews CG, Grant I (1987): Comparison of preferred and nonpreferred hand performance on four neuropsychological motor tasks. Clin Neuropsychol. 1: 324-334.

25. Schmidt SL, Oliveira RM, Rocha FR, Abreu-Villaca Y (2000): Influences of Handedness and Gender on the Grooved Pegboard Test. Brain Cogn. 44: 445-454.

26. Peters M, Servos P, Day R (1990): Marked sex differences on a fine motor skill task disappear when finger size is used as covariate. J Appl Psychol. 75: 87-90.

27. Peters M, Campagnaro P (1996): Do women really excel over men in manual dexterity? J Exp Psychol Hum Percept Perform. 22: 1107-1112.

28. Nicholson KG, Kimura D (1996): Sex Differences for Speech and Manual Skill. Percept Mot Skills. 82: 3-13.

29. Sella GE (2001): The hand grip: gender, dominance and age considerations. Eura Medicophys. 37: 161-170.

30. Boyd A, Golding J, Macleod J, Lawlor DA, Fraser A, Henderson J, et al. (2013): Cohort Profile: the 'children of the $90 \mathrm{~s}^{\prime}$--the index offspring of the Avon Longitudinal Study of Parents and Children. Int J Epidemiol, 2012/04/18. 42: 111-127.

31. Fraser A, Macdonald-Wallis C, Tilling K, Boyd A, Golding J, Davey Smith G, et al. (2013): Cohort Profile: The Avon Longitudinal Study of Parents and Children: ALSPAC mothers cohort. Int J Epidemiol. 42: 97-110.

32. Henderson SE, Sugden DA (1992): Movement Assessment Battery for Children manual. Sidcup: The Psychological Corporation.

33. Leask SJ, Crow TJ (2001): Word acquisition reflects lateralization of hand skill. Trends Cogn Sci. 5: 513-516.

34. Annett M (1985): Left, Right, Hand and Brain: The right Shift theory. London: Psychology Press.

35. McManus IC (1985): Handedness, language dominance and aphasia: a genetic model. Psychol Med Monogr Suppl, 1985/01/01. 8: 1-40.

36. RStudioTeam (2015): RStudio: Integrated Development for R. Boston, MA: RStudio, Inc.

37. Scerri TS, Brandler WM, Paracchini S, Morris AP, Ring SM, Richardson AJ, et al. (2011): PCSK6 is associated with handedness in individuals with dyslexia. Hum Mol Genet, 2010/11/06. 20: 608614.

38. Brandler WM, Morris AP, Evans DM, Scerri TS, Kemp JP, Timpson NJ, et al. (2013): Common variants in left/right asymmetry genes and pathways are associated with relative hand skill. PLoS Genet, 2013/09/27. 9: e1003751.

39. Francks C, Maegawa S, Lauren J, Abrahams BS, Velayos-Baeza A, Medland SE, et al. (2007): LRRTM1 on chromosome $2 \mathrm{p} 12$ is a maternally suppressed gene that is associated paternally with handedness and schizophrenia. Mol Psychiatry. 12: 1057,1129-1139. 
40. Ludwig KU, Mattheisen M, Muhleisen TW, Roeske D, Schmal C, Breuer R, et al. (2009): Supporting evidence for LRRTM1 imprinting effects in schizophrenia. Mol Psychiatry, 2009/07/25. 14: 743-745.

\section{Supplementary Figures}

Supplementary Figure S1. Distribution of laterality indexes. Histograms and Q-Q plots for a) PegQ; $b$ ) MarkQ; c) SortQ and d) GripQ. Each individual measure is represented by the green rugs at the bottom of each distribution. The blue lines describe normal distributions under the observed mean and variance. The Q-Q plots illustrate the differences between a normal (red lines) and the observed (black circles) distribution.

Supplementary Figure S2. Age effect on laterality indexes. The histograms on the left show the age, measured in days, when data were collected. The graph on the right plot the laterality indexes by age.

Supplementary Figure S3. Sex effect of correlation across indexes. Correlation of laterality measures in A) males and B) females. The cells along the diagonal show the empirical distribution for each index and the bar-plot for the preferred hand for writing. The boxes on the left of the main diagonal show the bivariate distribution of the indexes colour coded for preferred hand for writing (left = orange; right =blue). For example, the first box on the second raw of the matrix illustrates the PegQ scores on the $x$-axis and MarkQ on the $y$-axis. The bottom row shows the box-plots for each laterality index grouped by preferred hand for writing. The red lines are aligned along the zero for each index. The cells on the right of the diagonal show the correlation coefficients and the sample sizes from which they were calculated. The last column reports the confidence intervals for the box-plots shown in the bottom row, providing a measure on how the different indexes separate the right and left handers for preferred writing hand.

Supplementary Figure S4. Performance of both hands. The performance of both the right and left hand is plotted for each individuals in the four tasks: Pegboarg, Marking Squares, Sorting Matches and Grip strength. Equal performance of both hands would plot along the red line.

Supplementary Figure S5. Age effect on best performance. The best performance regardless of left or right preference was plotted against age for A) Pegboard, B) Marking squares, C) Sorting matches and D) Grip strength. Correlation and statistical significance are shown in red and a regression line is shown in blue. 